\title{
Anticoagulation related nephropathy
}

\author{
Mohamed E. Ibrahim, El-Metwally L. El-shehawy, Moaz A. Bakr, Ahmed E. Mansour
}

\begin{abstract}
Department of internal medicine, Benha Faculty of Medicine, Benha University, Egypt.

Correspondence to: Moaz A Bakr, Department of internal medicine, Benha Faculty of Medicine, Benha University, Egypt.
\end{abstract}

Email:

moazbakr333@gmail.com

Received: 19 July 2019

Accepted: 7 August 2020

\begin{abstract}
:
Background :Anticoagulation related nephropathy (ARN) is an under diagnosed complication of anticoagulation that is associated with increased renal morbidity and mortality. While warfarin has been in use since 1954, its harmful effects on the kidneys have only recently been fully recognized. ARN is clinically defined as acute kidney injury (AKI) without obvious aetiology in the setting of over anticoagulation. When it is caused by warfarin, it is often associated with supra therapeutic International Normalized Ratio (INR) (more than 3.0) .AIM: this work aimed to early detection of ARN and how to manage it. Patients and methods: This study was carried out in internal medicine department of Benha University Hospitals, where 50 patients on oral anticoagulation suffered from acute kidney injury were selected and subjected to careful history , clinical examination and laboratory investigation after taking their written consents from march 2019 to march 2020. Results: From the 50 patients ARN was observed in $30 \%$ of patients (15 patients) $.80 \%$ of patients were on warfarin and $20 \%$ of patients were on
\end{abstract} dapigatran. $34 \%$ of patients were hypertensive, $56 \%$ of patients were chronic kidney disease (CKD) and $40 \%$ of patients were diabetic .At the start of the study serum creatinine was $1.5 \pm 0.5$ $\mathrm{mg} / \mathrm{dl}$ and mean INR was $2.4 \pm 0.6$ and after starting oral anticoagulants (OACs) mean INR increased to $6.3 \pm 1.8$ and serum creatinine increased to $2.6 \pm 0.8 \mathrm{mg} / \mathrm{dl}$ at the first month of follow up then by controlling INR to $2.8 \pm 0.4$ at the second month serum creatinine decreased to $2 \pm 0.6$ mg/dl. Conclusion: ANR should be suspected in any patients with over anticoagulation and increased serum creatinine and this need rapid intervention to reverse renal injury.

Keywords: Anticoagulation related nephropathy, acute kidney injury, chronic kidney disease , dapigatran related nephropathy, warfarin related nephropathy. 



\section{Introduction}

The incidence of ARN is difficult to determine due to its changing definition and diagnostic criteria.ARN was a pathologic diagnosis defined with dysmorphic red blood cells (RBCs) implying injury to the glomerular filtration barrier, uniformhaemorrhage through all fields as to exclude biopsy artefact, the presence of obstructive tubular RBC casts, and absence of glomerulonephritis or other inflammatory changes that could account for glomerular haemorrhage[1]

Now, most cases of ARN are not biopsyproven and it should be considered in the differential diagnosis of any patient on anticoagulation presenting with AKI especially in the setting of supra therapeutic INR or excessive direct oral anticoagulants (DOAC) dose [2]. The initial work-up should consist of a clinical exam, careful medication history and urinalysis to assess for hematuria and subsequent diagnostic tests which may be needed to exclude other causes of AKI or confirm the diagnosis in high risk individuals [3].

For patients on warfarin and DOACs, kidney function should be monitored regularly throughout treatment and with increased frequency during the first
3 months with maintain INR at target level [4]. But even if the renal function returns to baseline following the ARN episode, some of the renal tubules will have been destroyed by the obstructive RBCs casts thus permanently decreasing the nephron mass of the kidney. The tubules that do survive the ischemic and oxidative insult will likely manifest hyper filtration injury that leads to accelerated CKD progression [5]

\section{Patients and methods}

This is a cohort prospective study that was carried out on 50 patients in Benha university hospitals internal medicine department from March 2019 to March 2020. Ethical approval was obtained by themedical ethical committee of Benha faculty of medicine and written consent was taken.

\section{Inclusion criteria:}

1) age $>18$ years old

2) INR >3

3) Increase in creatinine level more than $0.3 \mathrm{mg} / \mathrm{dl}$ or increase of creatinine level $>50 \%$ from baseline 


\section{Exclusion criteria:}

1) age $<18$ years old

2) INR $<3$

3) increase in creatinine level less than $0.3 \mathrm{mg} / \mathrm{dl}$ or increase of creatinine level < $50 \%$ from baseline.

\section{Methods}

History taking to identifying patients with risk factors of $\mathrm{ARN}$ as $\mathrm{CKD}$, age, diabetes mellitus, and hypertension, clinical examination, laboratory investigation as complete blood picture(CBC), Serum creatinine, Blood urea nitrogen (BUN), Electrolytes, Serum Albumin, Prothrombin time (PT), INR and Urinalysis: focusing on Proteinuria, hematuria and RBCs casts and Renal Ultrasound.

Frequency of renal function tests monitoring for patients on OACs after developing ARN: monthly in first 3 months then according to e-GFR:

e-GFR > 60 : every 6 months

e-GFR 30-60 : every 2 months

e-GFR $<30$ : every 2 months

Optimization of a patient's co-morbid conditions, such as diabetes mellitus and hypertension with either decreasing the dose or change the type of oral anticoagulant are the main lines of management of ARN.

\section{Statistical Analysis}

The collected data were revised, coded and tabulated using Statistical package for Social Science (IBM Corp. Released 2017. IBM SPSS Statistics for Windows, Version 25.0. Armonk, NY: IBM Corp.). Data were presented and suitable analysis was done according to the type of data obtained for each parameter.Shapiro test was done to test the normality of data distribution. Significant data was considered to be nonparametric.

\section{- Descriptive statistics:}

1. Mean, Standard deviation $( \pm \mathrm{SD})$ for parametric numerical data, while Median and range for non-parametric numerical data.

2. Frequency and percentage of nonnumerical data.

\section{- Analytical statistics:}

Student $\mathrm{T}$ Test was used to assess the statistical significance of the difference between two study group means. For the comparison of the three groups' means, one 
way analysis of variance (ANOVA) was used.

Chi-Square test was used to examine the relationship between two qualitative variables. Fisher's exact test: was used to examine the relationship between two qualitative variables when the expected count is less than 5 in more than $20 \%$ of cells. $\mathrm{p}$ is significant if $<0.05$ at confidence interval $95 \%$.

\section{Results:}

This study was conducted on 50 cases receiving OACs. Their mean age was 49 years, ranged from 19 to 64 years. They were 24 males (48\%) and 26 females $(52 \%)$.Forty cases received warfarin $(80 \%)$, while 10 cases received dapigatran (20\%).Thirty percent of all studied cases developed ARN (15 patients) 12 patients out of 40 patients received warfarin and 3 patients out of 10 patients received dapigatran). (Table 1)

Table (2), figure (1) showed that $30 \%$ of patients developed ARN in both who received warfarin or dapigatran .

Table (3) showed that mean RBC count in our study was 3.9X106/L, mean hemoglobin concentration was $11.2 \mathrm{~g} / \mathrm{dL}$, mean TLC was
8.1X109/L, mean platelet count was $318.8 X 109 / \mathrm{L}$, mean serum sodium was 137 , mean potassium was 4.3 , mean calcium was $8.6 \mathrm{mg} / \mathrm{dL}$, mean PO4 was $4.2 \mathrm{mg} / \mathrm{dL}$ mean PTH was 40.4, mean albumin was $4 \mathrm{~g} / \mathrm{dL}$, mean CRP was $10.3 \mathrm{mg} / \mathrm{L}$, mean RBCs count in urine was $21.9 \mathrm{RBCs} / \mathrm{HPF}, 56 \%$ had minimal proteinuria, $26 \%$ had RBCs casts. Also Mean serum creatinine was 1.5 $\mathrm{mg} / \mathrm{dL}$ when mean INR was 2.4 but at the end of the first month when mean INR was 6.3 , the mean serum creatinine increased to $2.6 \mathrm{mg} / \mathrm{dL}$, but with adjustment the dose of OACs mean INR was 2.8 by the end of the second month, thus mean serum creatinine improved to $2 \mathrm{mg} / \mathrm{dL}$ and to $1.6 \mathrm{mg} / \mathrm{dL}$ when mean INR was $2.7 \mathrm{By}$ the end of the third month. $36 \%$ of patients had eGFR> 60 , $48 \%$ hadeGFR between 60 and $30 \mathrm{ml} / \mathrm{min}$ and $16 \%$ had e-GFR less than $30 \mathrm{ml} / \mathrm{min}$ (table 3 ). Table (4) showed that no significant differences were found in laboratory data at the first month between those received warfarin and those received dapigatran. Also no significant difference was found in serum creatinine and INR at the second and the third months between those received warfarin and those received dapigatran. But e-GFR was significantly higher in those received dapigatran than those received warfarin (table 4) 

Table (5) revealed that all cases who developed ARN had underlying risk factors, compared to $62.9 \%$ in patients who did not develop ARN $(\mathrm{p}=0.005) . \quad \mathrm{DM}$ was significantly associated with ARN (73.3\% versus $25.7 \%, \mathrm{p}=0.002$ ). Hypertension and CKD did not differ significantly between both groups ( $p>0.05$ for each).CKD was the most common risk factor.(table 5). As shown in table (6), renal size did not differ significantly between those with and without ARN. However, echogenicity grades increased significantly with presence of ARN. (table 6)

Table (1). Demographic and clinical data distribution of all studied cases.

\begin{tabular}{lll}
\hline & & Cases $(\mathbf{N}=\mathbf{5 0})$ \\
\hline \multirow{2}{*}{ Age (years) } & Mean \pm SD & $49 \pm 9.3$ \\
& Range & $19-64$ \\
Males & $\mathbf{N}(\%)$ & $24(48 \%)$ \\
Females & $\mathbf{N}(\%)$ & $26(52 \%)$ \\
\hline
\end{tabular}

Table (2): Association of ARN with types of OACs.

\begin{tabular}{lccccccc}
\hline & Warfarin $(\mathbf{N}=\mathbf{4 0})$ & \multicolumn{2}{c}{ Dapigatran $(\mathbf{N}=\mathbf{1 0})$} & & OR & $\mathbf{9 5 \%} \mathbf{C I}$ \\
& $\mathbf{N}$ & $\mathbf{\%}$ & $\mathbf{N}$ & $\mathbf{\%}$ & & \\
\hline Non ARN & 28 & $70 \%$ & 7 & $70 \%$ & & & \\
ARN & 12 & $30 \%$ & 3 & $30 \%$ & & 1 & $0.220-4.536$ \\
\hline
\end{tabular}

OR, odds ratio, CI confidence interval, OACs : Oral anticoagulants, ARN : Anticoagulant Related Nephropathy.

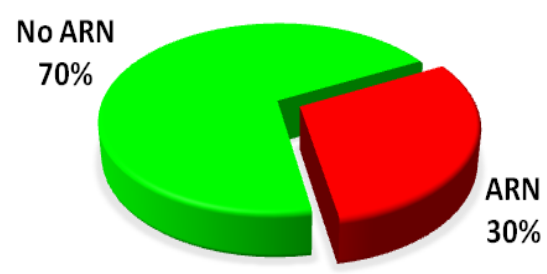

Figure (1): ARN in all studied cases 
Table (3): Baseline laboratory data of all patients

\begin{tabular}{lllll}
\hline & & N=50 & & \\
\hline Serum creatinine $(\mathbf{m g} / \mathbf{d L})$ & mean \pm SD & 1.5 & \pm & 0.5 \\
INR & mean \pm SD & 2.4 & \pm & 0.6 \\
RBCs $(\mathbf{X 1 0} / \mathbf{L})$ & mean \pm SD & 3.9 & \pm & 0.3 \\
Hemoglobin $(\mathbf{g} / \mathbf{d L})$ & mean \pm SD & 11.2 & \pm & 1 \\
TLC $\left(\mathbf{X} 1 \mathbf{9}^{\mathbf{9}} / \mathbf{L}\right)$ & mean \pm SD & 8.1 & \pm & 2.2 \\
Platelet $(\mathbf{X 1 0} / \mathbf{L})$ & mean \pm SD & 318.8 & \pm & 81.4 \\
Serum Na(mmol/L) & mean \pm SD & 137 & \pm & 4.7 \\
Serum K(meq/L) & mean \pm SD & 4.3 & \pm & 0.6 \\
Calcium $(\mathbf{m g} / \mathbf{d L})$ & mean \pm SD & 8.6 & \pm & 0.2 \\
PO4 $(\mathbf{m g} / \mathbf{d l})$ & mean \pm SD & 4.2 & \pm & 0.5 \\
PTH $(\mathbf{p g} / \mathbf{m L})$ & mean \pm SD & 40.4 & \pm & 10.1 \\
Albumin $(\mathbf{g} / \mathbf{d L})$ & mean \pm SD & 4 & \pm & 0.4 \\
CRP $(\mathbf{m g} / \mathbf{L})$ & mean \pm SD & 10.3 & \pm & 3.1 \\
RBCs in urine $(\mathbf{R B C s} / \mathbf{H P F})$ & mean \pm SD & 21.9 & \pm & 5 \\
Minimal proteinurea & $\mathbf{N}(\%)$ & 28 & & $56 \%$ \\
RBC casts & N $(\%)$ & 13 & & $26 \%$ \\
& & & & \\
\hline
\end{tabular}

Table (4): Laboratory data between those with and without ARN in first 3 months

\begin{tabular}{|c|c|c|c|c|c|c|c|c|}
\hline & \multirow{2}{*}{\multicolumn{2}{|c|}{ Serum creatinine $(\mathrm{mg} / \mathrm{dL})$}} & \multirow[b]{2}{*}{ mean \pm SD } & \multicolumn{2}{|c|}{ No ARN (N=35) } & \multicolumn{2}{|c|}{$\operatorname{ARN}(\mathbf{N}=15)$} & \multirow{2}{*}{$\begin{array}{l}\boldsymbol{P} \\
<0.001\end{array}$} \\
\hline \multirow{4}{*}{$\begin{array}{l}\text { First } \\
\text { month }\end{array}$} & & & & 1.1 & \pm 0.3 & 6.2 & \pm 2.0 & \\
\hline & \multirow{2}{*}{\multicolumn{2}{|c|}{$\begin{array}{l}\text { Serum creatinine change }(\mathrm{mg} / \mathrm{dL}) \\
\text { Blood urea }(\mathrm{mg} / \mathrm{dL})\end{array}$}} & mean \pm SD & -0.03 & \pm 0.01 & 4 & \pm 1.3 & $<0.001$ \\
\hline & & & mean \pm SD & 32.2 & \pm 8.6 & 68.6 & \pm 22.6 & $<0.001$ \\
\hline & \multicolumn{2}{|c|}{ PT (second) } & $\operatorname{mean} \pm \mathrm{SD}$ & 39.6 & \pm 7.3 & 56.5 & \pm 15.3 & $<0.001$ \\
\hline & \multicolumn{2}{|l|}{ INR } & mean \pm SD & 5.6 & \pm 1 & 8 & \pm 2.2 & $<0.001$ \\
\hline Second & \multicolumn{2}{|c|}{ Serum creatinine $(\mathrm{mg} / \mathrm{dL})$} & $\operatorname{mean} \pm \mathrm{SD}$ & 1.1 & \pm 0.3 & 3.9 & \pm 1.1 & $<0.001$ \\
\hline month & \multicolumn{2}{|l|}{ INR } & mean $\pm S D$ & 2.8 & \pm 0.4 & 3.0 & \pm 0.3 & 0.115 \\
\hline Third & \multicolumn{2}{|c|}{ Serum creatinine $(\mathrm{mg} / \mathrm{dL})$} & $\operatorname{mean} \pm \mathrm{SD}$ & 1.1 & \pm 0.3 & 2.8 & \pm 0.9 & $<0.001$ \\
\hline \multirow[t]{5}{*}{ month } & \multirow{5}{*}{$\begin{array}{l}\text { INR } \\
\text { eGFR } \\
\text { (ml/mint) }\end{array}$} & & $\operatorname{mean} \pm \mathrm{SD}$ & 2.7 & \pm 0.2 & 2.7 & \pm 0.2 & 0.593 \\
\hline & & & mean \pm SD & 79.5 & \pm 25.9 & 29.7 & \pm 14.8 & $<0.001$ \\
\hline & & eGFR > 60 & $\mathbf{N}, \%$ & 18 & $51.4 \%$ & 0 & $0 \%$ & \\
\hline & & eGFR 30-60 & $\mathbf{N}, \%$ & 17 & $48.6 \%$ & 7 & $46.7 \%$ & $<0.001$ \\
\hline & & eGFR $<30$ & $\mathrm{~N}, \%$ & 0 & $0 \%$ & 8 & $53.3 \%$ & \\
\hline
\end{tabular}


Table (5): Comparison of risk factors between patients with and without ARN.

\begin{tabular}{|c|c|c|c|c|c|}
\hline & \multicolumn{2}{|c|}{ No ARN (N=35) } & \multicolumn{2}{|c|}{$\operatorname{ARN}(\mathbf{N}=15)$} & \multirow{2}{*}{$\boldsymbol{P}$} \\
\hline & $\mathbf{N}$ & $\%$ & $\mathbf{N}$ & $\%$ & \\
\hline No risk factors & 13 & $37.1 \%$ & 0 & $0 \%$ & \multirow{2}{*}{0.005} \\
\hline Presence of risk factors & 22 & $62.9 \%$ & 15 & $100 \%$ & \\
\hline HTN & 12 & $34.3 \%$ & 5 & $33.3 \%$ & 0.948 \\
\hline CKD & 18 & $51.4 \%$ & 10 & $66.7 \%$ & 0.320 \\
\hline DM & 9 & $25.7 \%$ & 11 & $73.3 \%$ & 0.002 \\
\hline
\end{tabular}

ARN : Anticoagulant Related Nephropathy, HTN : Hypertension, CKD : chronic kidney disease., DM : Diabetus Mellitus

Table (6): Comparison of renal ultrasound findings between those with and without ARN.

\begin{tabular}{|c|c|c|c|c|c|c|c|}
\hline \multirow[b]{2}{*}{ Renal size $(\mathbf{c m})$} & & \multirow[b]{2}{*}{ mean \pm SD } & \multicolumn{2}{|c|}{ No ARN (N=35) } & \multicolumn{2}{|c|}{ ARN (N=15) } & \multirow{2}{*}{$\begin{array}{l}\boldsymbol{P} \\
0.210\end{array}$} \\
\hline & & & 10.1 & \pm 0.9 & 9.8 & \pm 0.5 & \\
\hline \multirow[t]{3}{*}{ Echogenicity } & Normal & $\mathrm{N}, \%$ & 21 & $60 \%$ & 3 & $20 \%$ & \\
\hline & grade I echogenicity & $\mathrm{N}, \%$ & 8 & $22.9 \%$ & 5 & $33.3 \%$ & 0.024 \\
\hline & grade II echogenicity & $\mathrm{N}, \%$ & 6 & $17.1 \%$ & 7 & $46.7 \%$ & \\
\hline
\end{tabular}

\section{Discussion}

Anticoagulant therapy is essential to evade thrombo-embolic events as in patients with atrial fibrillation or deep vein thrombosis. Currently, DOACs are being rapidly used owing to their safety, efficacy, and convenience.[6]

ARN is a clinical syndrome of AKI due to glomerular haemorrhage, tubular obstruction and tubular injury and historically it was described in patients with supra therapeutic levels of warfarin
[7].ARN is a complication associated with the use of anticoagulants that has been reported in recent years, especially in cases involving warfarin-related nephropathy (WRN).[8]

This condition is frequently underdiagnosed and is characterized by the following criteria, which may not occur together:-

- excessive anticoagulation as INR $>3$ in most studies. 
- Haematuria

- ARF unexplained by other causes (worsening of baseline creatinine by $>0.3$ $\mathrm{mg} / \mathrm{dL})$.[9]

Identified characteristic features of ARN in renal biopsies, including acute kidney injury showed presence of red blood cells in Bowman's space and renal tubular obstruction by red blood cell casts. Moreover, the reported rate of $\mathrm{ARN}$ is higher in patients affected by CKD, which might be a risk factor for ARN [2]

ARN just might be the most common kidney problem that clinicians have never seen. ARN is a recently discovered as a cause of AKI and possibly, progressive of CKD as well. [10]

In our study, we aimed to study the incidence of ARN, how to detect it early to decrease its complication .

Regarding to demographic data, our study included 50 patients receiving OACs. Their mean age was 49 years, ranged from 19 to 64 years. They were 24 males $(48 \%)$ and 26 females $(52 \%)$. There was no significant difference regarding age and gender between those with and without WRN and DRN ( $\mathrm{P}=0.09,0.56$ respectively $)$ and this was in agreement with study done in 2019 which showed that no age, sex or racial difference was found between those with and without ARN.[11]

Forty cases received warfarin $(80 \%)$, while 10 cases received dapigatran (20\%). Warfarin has been the only available oral anticoagulant for more than half a century. It is estimated that more than 30 million warfarin prescriptions are filled annually in the USA .[12]

NOACs are being prescribed increasingly as they do not require routine coagulation monitoring and have improved clinical outcomes overall including decreased risk of major bleeding, making them particularly attractive alternatives to warfarin [13]. Although all of these advantages of NOAC over Warfarin, warfarin still used more frequently may be due to economic issues.

In our study $30 \%$ of those received warfarin developed ARN. The same frequency was obtained with those received dapigatran (30\%), with no significant differences between both OACs regarding ARN development and this was concordant with [14] who reported that Prevalence of ARN ranged from $19 \%$ to $63 \%$ among the four included cohort studies. Fifty patients (100\%) developed over anticoagulation with a median INR value of 6.06 (range: 3.9-12). 
Only 15 of these 50 patients (30\%) develop AKI with a median Serum creatinine 5 $\mathrm{mg} / \mathrm{dl}$ (range : $2.8-11.8 \mathrm{mg} / \mathrm{dl}$ ). This was supported by study done in 2017 which said that eleven patients (84\%) developed over anticoagulation with a median INR value of 3.8 (range: $2.51-10$ ) with only 4 of these 11 patients $(36 \%)$ developed AKI with a median serum creatinine during AKI of 7.83 mg/dl (range: $3.51-18.71 \mathrm{mg} / \mathrm{dl})$. [15]

Once patients developed AKI after over anticoagulation, we stopped the anticoagulant to return the INR to the target below 3 and follow up the patients and Kidney function tests for the first 3 months. This was supported by an experimental study that found the same results and found that tubular toxicity by heme-induced oxidative stress is the main mechanism of AKI. Indeed, in rats with 5/6 nephrectomy and warfarin induced overanticoagulation, treatment with the antioxidant as $\mathrm{N}$ AcetylCysteine prevents AKI without any effect on hematuria or tubular RBC development. [16]

Two patient rapidly recovered renal function in second month and returned to baseline in third month with Serum Creatinine $1.1 \mathrm{mg} / \mathrm{dl}(\mathrm{e}-\mathrm{GFR}=66,65 \mathrm{~mL} /$ $\min / 1.73 \mathrm{~m} 2$ ), Five patients recovered their baseline serum creatinine after 3 months ranging (1.9-2.8 mg/dl ), Six patients showed partial recovery after 3 months with Serum creatinine ranging (1.8$3.2 \mathrm{mg} / \mathrm{dl} \mathrm{),} \mathrm{two} \mathrm{of} \mathrm{them} \mathrm{required} \mathrm{dialysis}$ therapy for 5 sessions and Two patients required dialysis till the last follow up (eGFR $=8,10 \mathrm{~mL} / \min / 1.73 \mathrm{~m} 2$ )respectively.

At the 9 month follow up visit, two patients had stage 2 CKD (e-GFR > 60 ), five patients had stage $3 \mathrm{CKD}$ (median eGFR $=41 \mathrm{~mL} / \min / 1.73 \mathrm{~m} 2$ ) and Six patients had stage 4 CKD (median e-GFR $=21.5 \mathrm{~mL} / \mathrm{min} / 1.73 \mathrm{~m} 2$ ).

The outcome was recovery in $46.7 \%$, partial recovery in $40 \%$ and dialysis in $13.3 \%$. Although both warfarin and dapigatran had the same ARN frequency, DRN had better e-GFR than WRNs. Outcome was better in DRN, although did not differ significantly when compared to WRN. This may be attributed to low sample size.

This was supported by study done in 2009 which showed a report of 9 cases; 4 of them showed recovery and dialysis was needed in 4 cases [1]. Another study added that patients with ARN have had various degrees of renal recovery. Some can achieve normal kidney function, though, 
unfortunately, most patients require hemodialysis. But one study reported that as many as $66 \%$ were dialysis dependent. [17]

As regarding risk factors between those with and without ARN we found that all cases who developed ARN had underlying risk factors, compared to $62.9 \%$ in patients who did not develop ARN ( $p=0.005)$. DM was significantly associated with ARN (73.3\% versus $25.7 \%, \quad \mathrm{p}=0.002)$. Hypertension and CKD did not differ significantly between both groups $(p>0.05$ for each).Risk factors did not differ significantly between WRN and DRN ( $>0.05$ for each).CKD was the most common risk factor in general.

This was supported by study done in 2018 which found that moderate or severe coagulopathy induced by warfarin or other anticoagulants, especially if INR $>4$ and $\mathrm{CKD}$, is the strongest risk factor for ARN, and in these cases, prognosis is also worse than those without CKD [18]. Other independent predictors of AKI risk in these patients were age, diabetes mellitus, heart failure, hypertension, and glomerulonephritis particularly with nephrotic syndrome. Another study done in 2016 also found that CKD is the strongest risk factor for ARN (33-37\% incidence in patients with CKD versus $16.5 \%$ in those without) [2].

In our study among ARN patients, indications of OACs was AF in $66.7 \%$ and DVT in $33.3 \%$ and this was supported with study done in 2018 which showed that the main indications for OACs are atrial fibrillation, venous thromboembolism, and status post heart valve replacement [19].

According to laboratory data, there was statistical correlation between ARN and RBCs in urine and presence of RBCs casts $(\mathrm{P}<0.001)$ and this correlation was significant; patients receiving OACs who developed ARN had higher urine RBCs and RBCs casts when compared to those who did not develop ARN and this agreed with study done in 2019 which cleared that RBCs casts in the tubules in renal biopsy and RBCs casts in urine sediment in ARN patients [1].

According to renal ultrasound, renal size did not differ significantly between those with and without ARN $(\mathrm{P}=0.2)$. However, echogenicity grade increased significantly with presence of $\mathrm{ARN}(\mathrm{P}=0.024)$ and this agreed with a case report which founded bilateral mild increase in renal parenchymal echogenicity [20]. 


\section{Conclusion}

OACs are widely used drugs in very dangerous situations, adjustment of the drug dose is very important to avoid renal injury and flow up of renal function tests is mandatory if OACs are indicated.ARNappears to be more appropriate than WRN, as any OACs can cause ARN.

\section{Abbreviation}

ARN (anticoagulant related nephropathy)

OACs (oral anticoagulants)

WRN (warfarin related nephropathy)

DRN (dapigatran related nephropathy)

CKD (chronic kidney disease)

INR (international normalization ratio)

$\mathrm{AF}$ (atrial fibrillation)

DVT (deep venous thrombosis)

e-GFR(estimated glomerular filtration rate)

DOACs (direct oral anticoagulants)

NOACs(new oral anticoagulant)

ARF (acute renal failure)

AKI(acute kidney injury)

\section{Acknowledgment}

The authors would like to acknowledge the clinical pathologists, technicians and other medical staffs for their support in achievement of this study.

\section{References:}

1)BrodskySV,SatoskarA,ChenJ,NadasdyG,EagenJW, HamiraniM,etal(2009):Acutekidneyinjuryduring warfarin therapy associated with obstructive tubular red blood cellcasts:areportof9cases. Am J Kidney Dis 54:1121-1126,

2)Wheeler DS, Giugliano RP and Rangaswami J. (2016) Anticoagulation-related nephropathy.J ThrombHaemost; 14: 461-467

3)Christiaans SC, Wagener BM, Esmon CT and Pittet JF. (2013) Protein C and acute inflammation: a clinical and biological perspective. Am J Physiol Lung Cell Mol Physiol.;305 (7):L455-66..

4)Böhm, M., Ezekowitz, M. D., Connolly, S. J., Eikelboom, J. W., Hohnloser, S. H., Reilly, P. A. et al (2015). Changes in renal function in patients with atrial fibrillation: an analysis from the RE-LY trial. Journal of the American College of Cardiology, 65(23), 2481-2493.

5)Venkatachalam MA,Griffin KA , lan R, Geng H ,SaikumarP,Bidani AK( 2010 ): acute kidney injury: a springboard for progreesion in chronic kidney disease.Am $\mathrm{J}$ physiol renal physiol.;298(5)F1078-94.

6)Fujino Y, Takahashi C, Mitsumoto K and Uzu T. (2019):Rivaroxaban-related acute kidney injury in a patient with $\operatorname{IgA}$ vasculitis. BMJ Case Rep.. https ://doi.org/10.1136/bcr-2018-22775 6.

7)Zhang, C., Gu, Z. C., Ding, Z., Shen, L., Pan, M. M., Zheng, Y. L. et al . (2019). Decreased risk of renal impairment in atrial fibrillation patients receiving non-vitamin $\mathrm{K}$ antagonist oral anticoagulants: A pooled analysis of randomized controlled trials and real-world studies. Thrombosis Research, 174, 16-23. 
Benha medical journal vol. 37, special issue (Internal medicine and Hepatology), 2020

8)Wells, G. A., Shea, B., O'connell, D., Peterson, J.,

Welch, V., Losos, M., et al . (2016). The

Newcastle-Ottawa Scale (NOS) for assessing the quality if nonrandomized studies in meta-analyses.

2009. Epub Available from: URL:

http://www.ohri.ca/programs/clinical_epidemiology /oxford. htm [cited 2009 Oct 19].

9)Brodsky SV( 2014):Anticoagulants and acute kidney injury: clinicalandpathology.Kidney Res ClinPract; 33: 174-180

10) Jansky L, Mukkamala P, Jebakumar D, Rao A,Goldson TM and Forjuoh SN(2018):Acute kidney injuryand undiagnosed immunoglobulin A ne-phropathy after dabigatran therapy.ProcBaylUniv Med Cent31: 321-323,

11) RichardJ. Glassock(2019):CJASN Jun, 14 ( 6) 935937 ; DOI: 10.2215/CJN.02470319

12) Brodsky SV, Nadasdy $T$, Rovin $B H$, Satoskar AA, Nadasdy GM, Wu HM, et al.( 2011) :Warfarin-related nephropathy occurs in patients with and without chronic kidney disease and is associated with an increased mortality rate. Kidney Int.;80(2):181-9.

13) Bacchus F and Schulman S.(2015):Clinical experience with the new oral anticoagulants for treatment of venous thromboembolism. Arterioscler Thromb Vasc Biol.;35(3):513-9.

14) Moura, K. B. D. A., Behrens, P. M. P., Pirolli, R., Sauer, A. B., Sandoval, D. E. M., Veronese, et al . (2019). Anticoagulant-related nephropathy: systematic review and metaanalysis. Clinical Kidney Journal. Oxford. Vol. 12, no. 3 (2019), p. 400-407.
15) Golbin L, Vigneau C, Touchard G, Thervet E, Halimi JM, Sawadogo T, et al.(2017):Warfarinrelated nephropathy induced by three different vitamin $\mathrm{K}$ antagonists: analysis of 13 biopsy-proven cases. Clin Kidney J. Jun;10(3):381-388. doi: 10.1093/ckj/sfw133. Epub 2017 Feb 8. PMID: 28616216; PMCID: PMC5466118.

16) Ware, K., Qamri, Z., Ozcan, A., Satoskar, A. A., Nadasdy, G., Rovin, B. H. et al . (2013). Nacetylcysteine ameliorates acute kidney injury but not glomerular hemorrhage in an animal model of warfarin-related nephropathy. American Journal of physiology-Renal physiology, 304(12), F1421F1427.

17) Thomas oliver, liann Abu salman, BrandonCiaudelli, David A Cohen and Wynnwood (2019):anticoagulant related nephropathy the most common diagnosis you never heard of the American journal of medicine vol 132,No 8, august

18) AnveshGolla, R. Goli, V. K. Nagalla, B. V. Kiran, D. S. B. Raju and M. S. Uppin (2018): Warfarin-related Nephropathy Indian J Nephrol. Sep-Oct; 28(5): 378-381. doi: 10.4103/ijn.IJN/3/17.

19) Altiok E and Marx N. (2018):Oral Anticoagulation. DtschArztebl Int. Nov 16;115(46):776-783.

doi:

10.3238/arztebl.2018.0776. PMID: 30602410; PMCID: PMC6329367.

20) Nazia sharfuddin , Mahra

Nourbakhsh, Alan

Box, Hallgrimur

Benediktsson, and Daniel

A. Muruve

(2018):Anticoagulant related nephropathy induced by dabigatran ";case report in nephrology vol7381505.9dec2018, doi10.1155/2018/73815

To cite this article: Mohamed E. Ibrahim, El-Metwally L. El-shehawy, Moaz A. Bakr, Ahmed E. Mansour. Anticoagulation related nephropathy. BMFJ 2020; 37 (internal medicine and Hepatology): 99-110. DOI: 10.21608/bmfj.2020.36439.1293 
Original article

DOI: 10.21608/bmfj.2020.25062.1224 